\title{
Electroacupuncture Inhibits the Activation of p38MAPK in the Central Descending Facilitatory Pathway in Rats with Inflammatory Pain
}

\author{
Man-Li Hu, ${ }^{1}$ Fei-Yan Zhou, ${ }^{1}$ Jing-Jing Liu, ${ }^{1}$ Yi Ding, ${ }^{1}$ \\ Ju-Ming Zhong, ${ }^{2}$ and Ming-Xing Ding ${ }^{1}$ \\ ${ }^{1}$ College of Veterinary Medicine, Huazhong Agricultural University, Wuhan 430070, China \\ ${ }^{2}$ College of Veterinary Medicine, Auburn University, 212 Greene Hall, Auburn, AL 36849-5518, USA \\ Correspondence should be addressed to Ming-Xing Ding; dmx@mail.hzau.edu.cn
}

Received 6 July 2017; Revised 15 September 2017; Accepted 1 November 2017; Published 22 November 2017

Academic Editor: Jian Kong

Copyright (c) 2017 Man-Li Hu et al. This is an open access article distributed under the Creative Commons Attribution License, which permits unrestricted use, distribution, and reproduction in any medium, provided the original work is properly cited.

The mitogen-activated protein kinases (MAPKs), especially p38MAPK, play a pivotal role in chronic pain. Electroacupuncture (EA) relieves inflammatory pain underlying the descending pathway, that is, the periaqueductal gray (PAG), the rostral ventromedial medulla (RVM), and the spinal cord dorsal horn (SCDH). However, whether EA antagonizes inflammatory pain through regulation of p38MAPK in this descending facilitatory pathway is unclear. Complete Freund's adjuvant (CFA) was injected into the hind paw of rats to establish inflammatory pain model. EA was administrated for $30 \mathrm{~min}$ at Zusanli and Kunlun acupoints at 0.5, 24.5, 48.5, and $72.5 \mathrm{~h}$, respectively. The paw withdrawal threshold (PWT), paw edema, and Phosphor-p38MAPK-Immunoreactivity (p-p38MAPKIR) cells were measured before $(0 \mathrm{~h})$ and at $1,3,5,7,25$, and $73 \mathrm{~h}$ after CFA or saline injection. EA increased PWT at 1, 3, 25, and $73 \mathrm{~h}$ and inhibited paw edema at 25 and $73 \mathrm{~h}$ after CFA injection. Moreover, the increasing number of p-p38MAPK-IR cells which was induced by CFA was suppressed by EA stimulation in PAG and RVM at 3 and $5 \mathrm{~h}$ and in SCDH at 5, 7, 25, and 73 h. These results suggest that EA suppresses inflammation-induced hyperalgesia probably through inhibiting p38MAPK activation in the descending facilitatory pathway.

\section{Introduction}

Chronic pain is maintained and modulated through nociceptive processes at spinal and supraspinal level. Electrophysiological and pharmacological researches have confirmed that stimulation of the periaqueductal gray (PAG) or the rostral ventromedial medulla (RVM) can influence spinal nociceptive process via inhibiting or facilitating nociceptive input [1-4]. Moreover, increased activity of peripheral nociception caused spinal sensitization and enhanced sensory information arriving at PAG and RVM, resulting in activation of descending facilitatory pathway [5-8]. Numerous studies document that the descending facilitation contributes to chronic pain states and maintenance of hyperalgesia $[5,7$, $9,10]$. Further, blocking the descending facilitatory pathway attenuates chronic pain and hyperalgesia $[5,11,12]$.
The activation of p38 mitogen-activated protein kinases (p38MAPK) signaling pathway plays a vital role in intracellular signal transduction on chronic pain [13-15]. Ni et al. [16] found that chronic constriction nerve injury induced mechanical hyperalgesia and increased expression of phosphorylated p38MAPK (p-p38MAPK) in the ventrolateral periaqueductal gray (vlPAG). Complete Freund's adjuvant (CFA) can induce inflammation pain and increase the number of Phosphor-p38MAPK-Immunoreactivity ( $\mathrm{p}$ p38MAPK-IR) cells in the RVM [17]. Studies have shown that $\mathrm{p}$-p38MAPK promoting the transcription factors (e.g., tumor necrosis factor $\alpha$, the activating transcription factor2) and inflammatory mediators (e.g., interleukin-1 and cyclooxygenase-2) in the spinal cord could result in pain facilitation $[18,19]$. Nevertheless, intrathecal injection or RVM microinjection of p38MAPK inhibitors decreases p38MAPK 
downstream propainful factors and alleviates hyperalgesia $[9,20,21]$, suggesting that inhibition of p38MAPK activation may attenuate inflammatory pain underlying the descending facilitatory pathway.

Electroacupuncture (EA) is a traditional complementary and alternative medicine approach with the advantages of safety, efficiency, and quantifiability to ameliorate inflammatory pain $[22,23]$. EA exerts effects against inflammatory pain, which has been confirmed by numerous clinical observations and studies [24-27]. Liang et al. reported that EA at bilateral Zusanli (ST36) and Kunlun (BL60) acupoints inhibited inflammatory-induced activation of spinal p38MAPK $[28,29]$. However, whether EA antagonizes inflammatory pain through regulation of $\mathrm{p} 38 \mathrm{MAPK}$ in the supraspinal levels is unclear.

In the present study, CFA-induced inflammatory pain model was applied to observe the effect of EA on the paw withdrawal threshold (PWT), paw volume, and the expression level of p-p38MAPK in PAG, RVM, and the spinal cord dorsal horn (SCDH). We hypothesize that EA could downregulate p-p38MAPK expression in the descending facilitatory pathway and then exert its inhibition function in the inflammatory pain.

\section{Materials and Methods}

2.1. Animal Preparation. Male Sprague-Dawley rats weighing $220 \pm 20 \mathrm{~g}$ were provided by Hubei Provincial Center for Disease Control and Prevention (number 42000600005600). One hundred and forty-four rats were housed six per cage with food pellets and water ad libitum and maintained on $12 \mathrm{~h}$ alternate light-dark cycles (7 a.m. to 7 p.m.). A quiet environment was provided, and the room temperature was maintained at $22 \pm 2^{\circ} \mathrm{C}$. Experimental animals were accustomed to being approached, acclimatized in individual plastic enclosures on a metal mesh and housing facilities (30 min/day) for one week before experiment. The experimental protocol was approved by the Animal Care Center, College of Veterinary Medicine, Huazhong Agricultural University (Wuhan, China).

\subsection{Establishment of Inflammation Model and Experimental} Groups. Inflammatory pain model was induced by injection of CFA (Sigma, USA) into the plantar surface of left hind paw. The rats were separated randomly into 4 groups (36 rats/group): (1) the saline group with saline injection (100 $\mu \mathrm{L}$ saline/per rat); (2) the CFA group with CFA injection (100 $\mu \mathrm{L} \mathrm{CFA/per} \mathrm{rat);} \mathrm{(3)} \mathrm{the} \mathrm{CFA} \mathrm{+} \mathrm{EA} \mathrm{group} \mathrm{with} \mathrm{CFA}$ injection (100 $\mu \mathrm{L} \mathrm{CFA/per} \mathrm{rat)} \mathrm{and} \mathrm{EA} \mathrm{treatment;} \mathrm{(4)} \mathrm{the} \mathrm{CFA}$ + sham group with CFA injection $(100 \mu \mathrm{L}$ CFA/per rat) and needle insertion without electrical stimulation. EA treatment was given at $0.5,24.5,48.5$, and $72.5 \mathrm{~h}$ after CFA or saline injection (Figure 1). To eliminate the stress effect, rats in saline group, CFA group, and CFA + sham group were immobilized the same as CFA + EA group. PWTs and paw volume of rats were measured before $(0 \mathrm{~h})$ and at $1,3,5,7,25$, and $73 \mathrm{~h}$ after CFA or saline injection (Figure 1). Six rats were taken from each group at $1,3,5,7,25$, and $73 \mathrm{~h}$, respectively. PWTs and paw volume were measured immediately and the

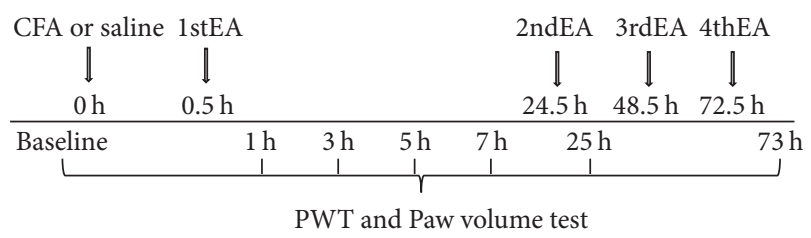

FIGURE 1: The timeline for the complete Freund adjuvant or saline injection, electroacupuncture treatment, the paw withdrawal threshold, and edema of the hind paw test.

rats were then euthanized for spinal and brain sampling. The expression of p-p38MAPK in the descending facilitatory pathway relevant areas of PAG, RVM, and SCDH was detected with immunohistochemistry.

2.3. Electroacupuncture. EA stimulation was conducted (9:00 a.m.), according to a previous report $[30,31]$. The sets of bilateral ST36 and BL60 which has shown a good effect on inflammatory pain were selected [28]. Four stainless steel acupuncture needles ( $0.30 \mathrm{~mm}$ in diameter, $13 \mathrm{~mm}$ in length) were inserted into bilateral ST36 $(4 \mathrm{~mm}$ lateral to the anterior tubercle of the tibia) and BL60 (at the ankle joint level and between the tip of the external malleolus and tendo calcaneus) acupoints. The needles in each set of EA groups were connected by a pair of wires to one output of WQ-6F Electronic Acupunctoscope (Beijing Xindonghua Electronic Instrument Co., Ltd., Beijing, China). The stimuli were set as square wave current output, with intensities in the range of 1-2 $\mathrm{mA}, 100 \mathrm{~Hz}$, and $2 \mathrm{~Hz}$ alternating frequencies (dense and disperse mode) for $30 \mathrm{~min}$ [32]. Throughout the EA, rats were kept in the housing facilities without anesthesia.

2.4. Behavioral Test. PWT was used to assess the inflammatory pain. Its assessment was conducted immediately before $(0 \mathrm{~h})$ and at $1,3,5,7,25$, and $73 \mathrm{~h}$ after CFA or saline injection. Rats were first acclimatized in individual plastic enclosures $\left(12 \times 22 \times 18 \mathrm{~cm}^{3}\right)$ on a metal mesh stand for $10 \mathrm{~min}$ before behavioral testing. The mechanical stimulus was delivered to the plantar surface of the left hind paw from the bottom floor of the plastic enclosures by an electronic von Frey anesthesiometer (ZS-Dichuang Science and Technology Development Co., Ltd., Beijing, China). A force transduction fitted with a $0.5 \mathrm{~mm}$ diameter polypropylene rigid tip was applied perpendicularly against the hind paw with an ever-increasing force from 0 to $50 \mathrm{~g}$ for a $20 \mathrm{~s}$ period. When the animal withdrew its hind paw, the mechanical stimulus was automatically stopped, and the force at which the animal withdrew its paw was recorded as PWT by the anesthesiometer. The procedure was repeated three times with $5 \mathrm{~min}$ interval. The percentage change in PWT was calculated as the following formula: $\Delta \%=\left(V_{n}-V_{0}\right) / V_{0} \times 100 \%$, where $V_{n}$ is the PTW after CFA or saline injection and $V_{0}$ is basal PWT.

2.5. Measurement of the Hind Paw Edema. The paw volume was assessed immediately after each PWT testing (the first test was regarded as basal paw volume) on left paw. A water displacement plethysmometer (YLS-7C, ZS-Dichuang 


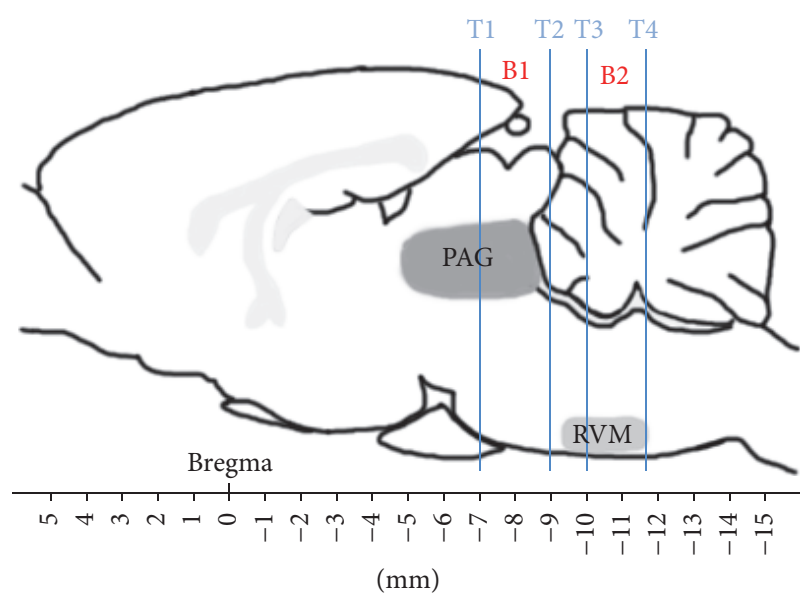

Figure 2: Brain sectioning. T1, T2, T3, and T4: marker tubes. T1, $\mathrm{T} 2$, T3, and T4 show transverse planes at $-7.00 \mathrm{~mm},-9.00 \mathrm{~mm}$, $-10.00 \mathrm{~mm}$, and $-11.60 \mathrm{~mm}$ rostral to the transverse plane of bregma, respectively. The locations of periaqueductal gray (PAG) and the rostral ventromedial medulla (RVM) in the brain blocks are presented. B1: a brain block containing PAG. B2: a brain block containing RVM.

Science and Technology Development Co., Ltd., Beijing, China) was used to measure the paw volume. The hind paw was immersed in a chamber containing distilled water up to the boundary between hairy and nonhairy skin, and the volume displacement was determined electronically. The percentage change in paw edema was calculated as the following formula: $\Delta \%=\left(T_{n}-T_{0}\right) / T_{0} \times 100 \%$, where $T_{n}$ is the paw volume after CFA or saline injection and $T_{0}$ is basal paw volume.

2.6. Immunohistochemistry. $\mathrm{SABC}$ immunohistochemistry was employed to detect the expression level of p-p38MAPK. Rats were deeply anesthetized with sodium pentobarbital $(80 \mathrm{mg} / \mathrm{kg}$, i.p.) and were perfused transcardially with $100 \mathrm{~mL}$ of saline followed by $500 \mathrm{~mL}$ of $4 \%$ paraformaldehyde in $0.1 \mathrm{~mol} / \mathrm{L}$ phosphate buffer at $\mathrm{pH}$ 7.4. Then, the heads of the rats were severed from the bodies and mounted in a stereotaxic instrument with the mouth bar set at the atlas standard $(-3.3 \mathrm{~mm})$ for blocking. According to the brain atlas of rats [33], four stainless steel marker tubes $(0.8 \mathrm{~mm}$ in diameter) were inserted perpendicularly into the brain along the midsagittal plane of the skull by means of stereotaxic electrode carriers at $-7.00 \mathrm{~mm},-9.00 \mathrm{~mm},-10.00 \mathrm{~mm}$, and $-11.60 \mathrm{~mm}$ from bregma, respectively (Figure 2 ). The brains were immersed in $4 \%$ paraformaldehyde for $48 \mathrm{~h}$ and then were removed from the skull and divided into several blocks according to the marker tubes. Two blocks (B1 and B2) and L4-L6 segments of spinal cord were obtained and embedded in paraffin. Each of the paraffin blocks was consecutively sectioned with a thickness of $5 \mu \mathrm{m}$. Four slides of the PAG, RVM, and SCDH were mounted on poly-lysine coated slides, deparaffinized, and rehydrated sequentially. Among these four slides, three of them were incubated with primary antibody solution containing p-p38MAPK antibody (1:1600,
CST, USA) while one was incubated with TBST instead of the antibody as negative control. The remaining experimental procedures of SABC immunohistochemistry were carried out according to the instructions (Wuhan Boster Biological Technology Ltd., Wuhan, China). The nucleus of positive cells was stained as brown yellow.

The gigantocellular reticular nucleus (Gi), the gigantocellular reticular nucleus pars alpha (GiA), and the nucleus raphe magnus (NRM) are main part of RVM. GiA and NRM were counted together because of the difficulty to distinguish them. Therefore, p-p38MAPK-IR cells of Gi and GiA + NRM were counted to represent those of RVM. Optical images of the stained nuclei were obtained under a microscope (Nikon ECLIPSE 80I, Nikon Corporation, and Tokyo, Japan) connected to a video-based and computer-linked system (highresolution pathological image analysis system-1000, Wuhan Qianping Ltd., Wuhan, China). Three slides of vlPAG, Gi, GiA + NRM, and SCDH were observed with 200x magnification and their locations were shown in Figure 3. The number of p-p38MAPK-IR cells on each nucleus was counted by the Image-Pro Plus 6.0 system (MediaCybernetics, Inc., Bethesda, MD, USA). The mean values calculated from each nucleus represented the $\mathrm{p}-\mathrm{p} 38 \mathrm{MAPK}$ per rat.

2.7. Statistical Analysis. PWT, paw volume, and the number of p-p38MAPK-IR cells were assessed or quantified by a skilled person who was blinded to the rat assignments. The results of PWT and paw volume were presented as the \% changes \pm SD. The results of the number of p-p38MAPKIR cells were presented as the mean $\pm \mathrm{SD}$. The data analysis was performed with SPSS 21.0 software (SPSS Inc., Chicago, USA). The percentage changes in PWT and paw volume were analyzed with repeated measures ANOVA. The number of p-p38MAPK-IR cells was assessed using one-way ANOVA. Bonferroni's post hoc test was applied when significant differences were found. $p<0.05$ was considered statistically significant.

\section{Results}

3.1. Effect of EA on Ipsilateral PWTs. The results showed that PWTs in saline group decreased slightly at $1-7 \mathrm{~h}$ and then recovered near baseline at 25 and $73 \mathrm{~h}$. PWTs of rats in CFA and CFA + EA groups were decreased at 1, 3, and $7 \mathrm{~h}$ obviously and reached the lowest level at $7 \mathrm{~h}$. PWTs of CFA or CFA + EA group were obviously lower $(p<0.01)$ than those of saline group at $1,3,5,7,25$, and 73 after CFA injection. However, PWTs of rats in CFA + EA group increased $(p<0.01)$ as compared with CFA at $1,3,25$, and $73 \mathrm{~h}$. No differences ( $p=1.000)$ were found between the CFA and the CFA + sham groups in PWTs (Figure 4).

3.2. Effect of EA on Ipsilateral Paw Edema. There were no changes of the paw volume in saline group at all measured points. The paw volume of CFA and CFA + EA groups increased gradually within $1-25 \mathrm{~h}$. The paw volume of CFA group was higher $(p<0.01)$ than that in saline group during the experiment. The paw volume of CFA + EA group was higher $(p<0.01)$ than that in saline group within $3-73 \mathrm{~h}$. 


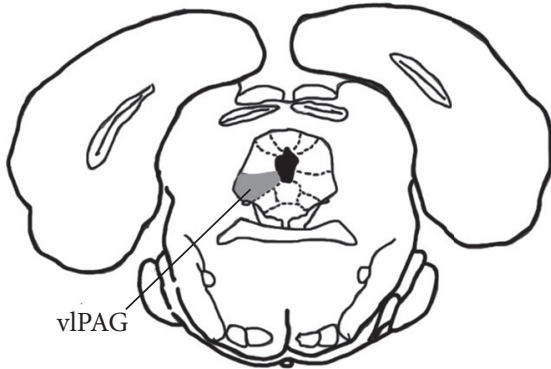

(a)

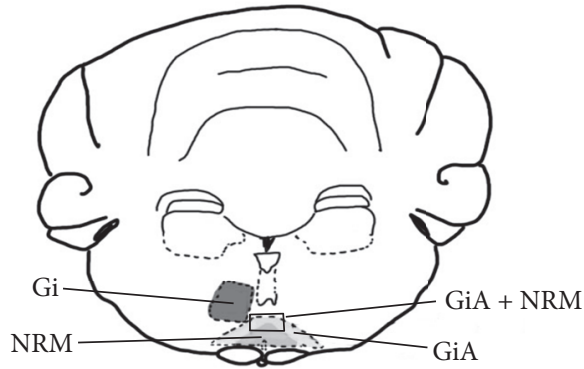

(b)

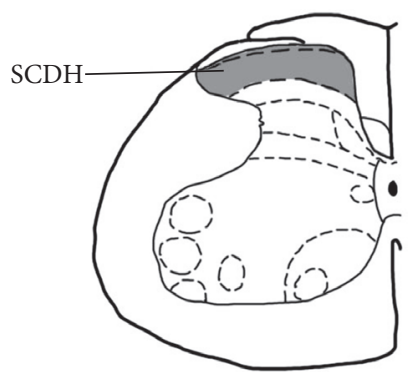

(c)

FIGURE 3: The nuclei (areas) locations used for the Phosphor-p38MAPK-Immunoreactivity cells counts. (a) The ventrolateral periaqueductal gray (vlPAG). (b) The gigantocellular reticular nucleus $(\mathrm{Gi})$ and the nucleus raphe magnus + gigantocellular reticular nucleus pars alpha (GiA + NRM). (c) The spinal cord dorsal horn (SCDH).

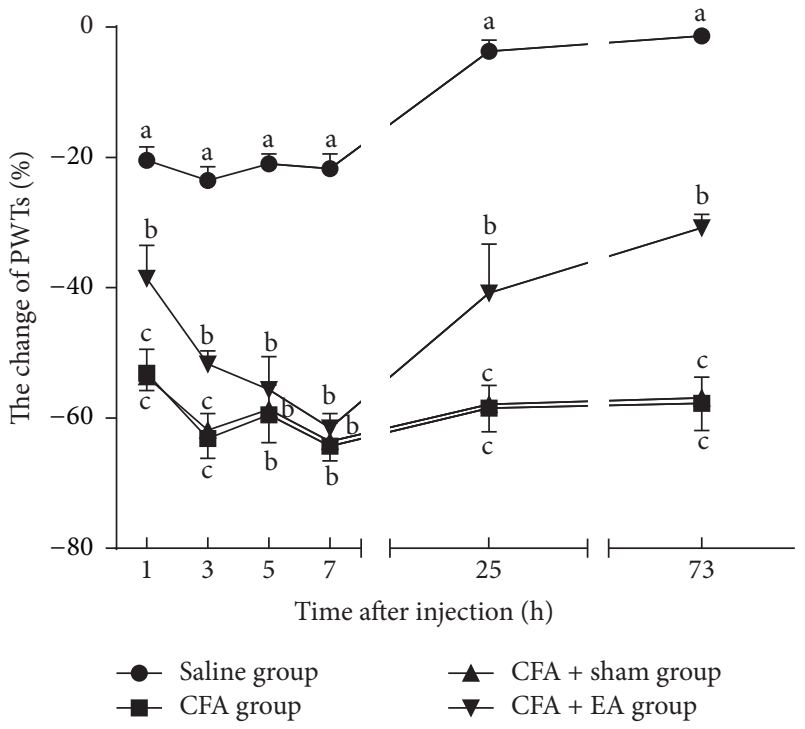

FIGURE 4: Effect of EA on ipsilateral PWTs at different time points. Values are mean $\pm \mathrm{SD}, \%, n=6$ /group. The values with different letters differ significantly in the same time point $(p<0.01)$.

The paw edema in CFA + EA group decreased $(p<0.05)$ at 25 and $73 \mathrm{~h}$ as compared with CFA group. No differences $(p=1.000)$ were detected between CFA and CFA + sham groups in the paw edema (Figure 5).

3.3. Phosphor-p38MAPK-Immunoreactivity. Phosphorp38MAPK-IR cells were observed in the vlPAG (Figure 6). Compared with saline group, CFA group induced an increase of p-p38MAPK-IR cells at $1-5 \mathrm{~h}(p<0.01)$, while CFA + EA group induced an increase of p-p38MAPK-IR cells at 1 and $5 \mathrm{~h}(p<0.05)$. After EA stimulation, p-p38MAPK-IR cells in CFA + EA group decreased $(p<0.01)$ as compared with CFA group at 3 and $5 \mathrm{~h}$. There was no difference $(p=1.000)$ in the number of p-p38MAPK-IR cells between CFA and CFA + sham groups.

The rostroventromedial medulla represented both $\mathrm{Gi}$ and GiA + NRM (Figure 7). Phosphor-p38MAPK-IR cells in CFA

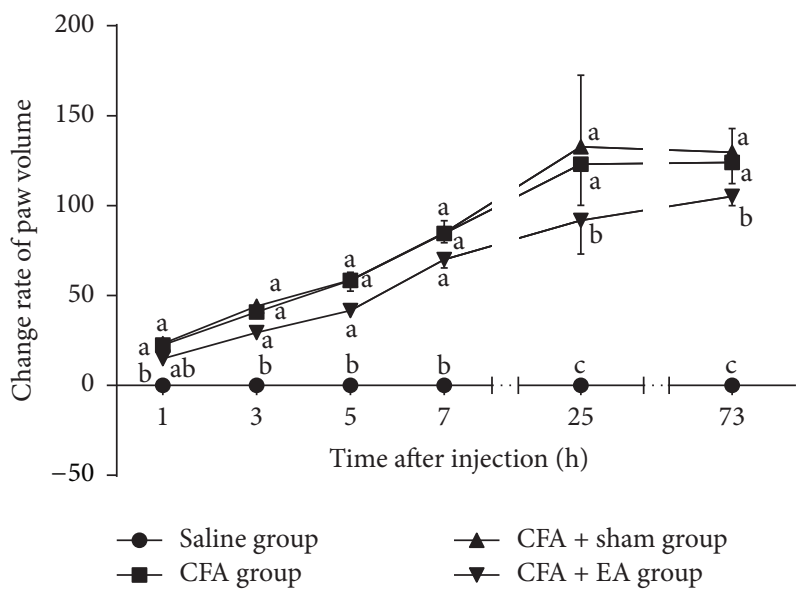

Figure 5: Effect of EA on ipsilateral paw edema at different time points. Values are mean $\pm \mathrm{SD}, \%, n=6$ /group. The values with different letters differ significantly in the same time point $(p<0.05)$.

group increased $(p<0.01)$ as compared with saline group in $\mathrm{Gi}$ at $1-7 \mathrm{~h}$ or $\mathrm{GiA}+\mathrm{NRM}$ at $1-5 \mathrm{~h}$. In CFA + EA group, p-p38MAPK cells increased $(p<0.01)$ more than saline group in Gi and GiA + NRM at 1-5 h. After EA stimulation, p-p38MAPK cells in CFA + EA group decreased $(p<0.01)$ as compared with CFA group in GI at 3 and $5 \mathrm{~h}$ or GiA + NRM at $1-5 \mathrm{~h}$. There was no difference $(p=1.000)$ in the number of p-p38-MAPK-IR cells between the CFA and CFA + sham groups.

Phosphor-p38MAPK-IR cells were observed in the SCDH (Figure 8). Compared with saline group, CFA group induced an increase $(p<0.01)$ in the number of $p$ p38MAPK-IR cells in the measured points. In CFA + EA group, p-p38MAPK cells increased $(p<0.05)$ more than that in saline group at $1,3,7,25$, and $73 \mathrm{~h}$. After EA stimulation, pp38MAPK-IR cells in CFA + EA group decreased $(p<0.05)$ as compared with CFA group at 5-73h. No differences $(p=$ 1.000) were detected between the CFA and the CFA + sham groups in the number of p-p38MAPK-IR cells. 

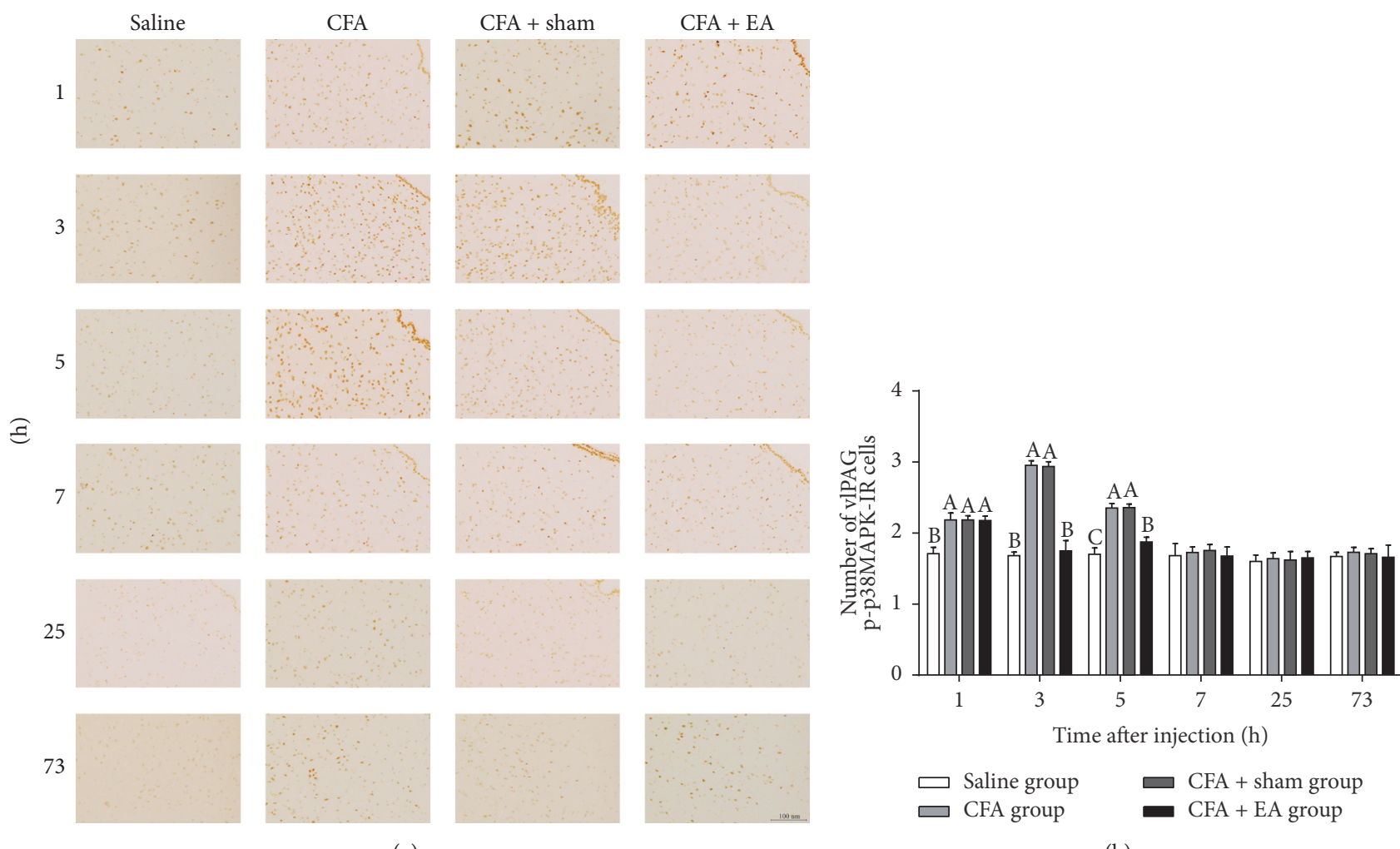

(a)

(b)

FIGURE 6: Integrated immunoreactivity analysis of p38 mitogen-activated protein kinase (p38MAPK) in the ventrolateral periaqueductal gray (vlPAG). (a) Immunohistochemical staining of Phosphor-p38MAPK-Immunoreactivity (p-p38MAPK-IR) cells in vlPAG was shown in saline, CFA, CFA + sham, and CFA + EA groups at different time points. (b) Quantification of p-p38MAPK-IR cells showing that EA suppressed expression of p-p38MAPK-IR cells in vlPAG. Values are mean $\pm \mathrm{SD}$, number $\times 10^{-2} /$ field, $n=6 /$ group. The values with different letters differ significantly in the same time point $(p<0.05)$. Scale bar represents $100 \mu \mathrm{m}$.

\section{Discussion}

CFA inflammation model was widely used for investigating the pain therapy. After the injection of CFA into paw, rats showed mechanical hyperalgesia and allodynia in $2 \mathrm{~min}$ and persisted for 7-14 d [34, 35], while paw edema started in $10 \mathrm{~min}$ and rose to peak at $3 \mathrm{~d}$ [35-38]. In the present study, the pain threshold was decreased at $1 \mathrm{~h}$ and still remained at a low level in $73 \mathrm{~h}$ after CFA injection, while CFAinduced inflammatory paw edema increased significantly and remained at high level during the whole experiment. These results showed the same tendency of inflammatory symptoms with previous studies.

Acupuncture therapy, especially EA, can induce potent analgesia to relieve persistent pain. Han [39] reported that frequency-dependent EA analgesia is mediated by the different opioid receptor subtypes. A frequency of $2 / 100 \mathrm{~Hz}$ EA simultaneously activating $\mu$-, $\delta$-, and $\kappa$-opioid receptors was reported to induce more effective analgesia than a single frequency $(2 \mathrm{~Hz}, 15 \mathrm{~Hz}$, or $100 \mathrm{~Hz})$ in rats [40]. Acupoints and EA times are also important factors associating with EA-induced anti-inflammatory pain effect. ST36 acupoint has been widely used to induce analgesic effect in rats in different pain model as well as BL60 acupoint [32, 41, 42]. In CFA model, EA applied at the bilateral ST36 and BL60 acupoints once per day could induce an increase in the PWTs without affecting the paw volume for 3 days [43]. Fang et al. [44] also reported that EA at ST36 and BL60 relieved CFA-induced inflammatory pain at 6,25 , and $49 \mathrm{~h}$ after CFA injection. Similar results were confirmed in a report that EA at ST36 and BL60 once per day could increase mechanical allodynia at 3 and $14 \mathrm{~d}$ and decreased paw edema at $14 \mathrm{~d}$ after CFA injection [28]. In the present study, the PWTs of CFA + EA group rats (receiving $2 \mathrm{~Hz}$ and $100 \mathrm{~Hz}$ alternating frequencies of EA at ST36 and BL60 acupoints for 30 min, once per day) increased gradually and were higher than CFA group at $1,3,25$, and $73 \mathrm{~h}$ after CFA injection. As EA repeated, its effect increased compared with CFA control group. Although EA lasted for $30 \mathrm{~min}$ each time, EA effect at $25 \mathrm{~h}$ was obviously higher than that at $5 \mathrm{~h}$ and was lower than that at $73 \mathrm{~h}$ within the EA group. These results suggested that EA-induced anti-inflammatory pain exhibited immediate and cumulative effects. In addition, the paw edema in CFA + EA group markedly decreased at 25 and $73 \mathrm{~h}$ as compared with CFA group $(p<0.05)$, indicating that EA exerts some therapeutic effect on inflammation.

Numerous studies indicated that p38MAPK participated in inflammatory response in central nerve system. In CFA model, the number of p-p38MAPK-IR cells was significantly increased in NRM for $0.5-1 \mathrm{~h}$ and in GiA at $0.5,3$, and $5 \mathrm{~h}$ [17]. 


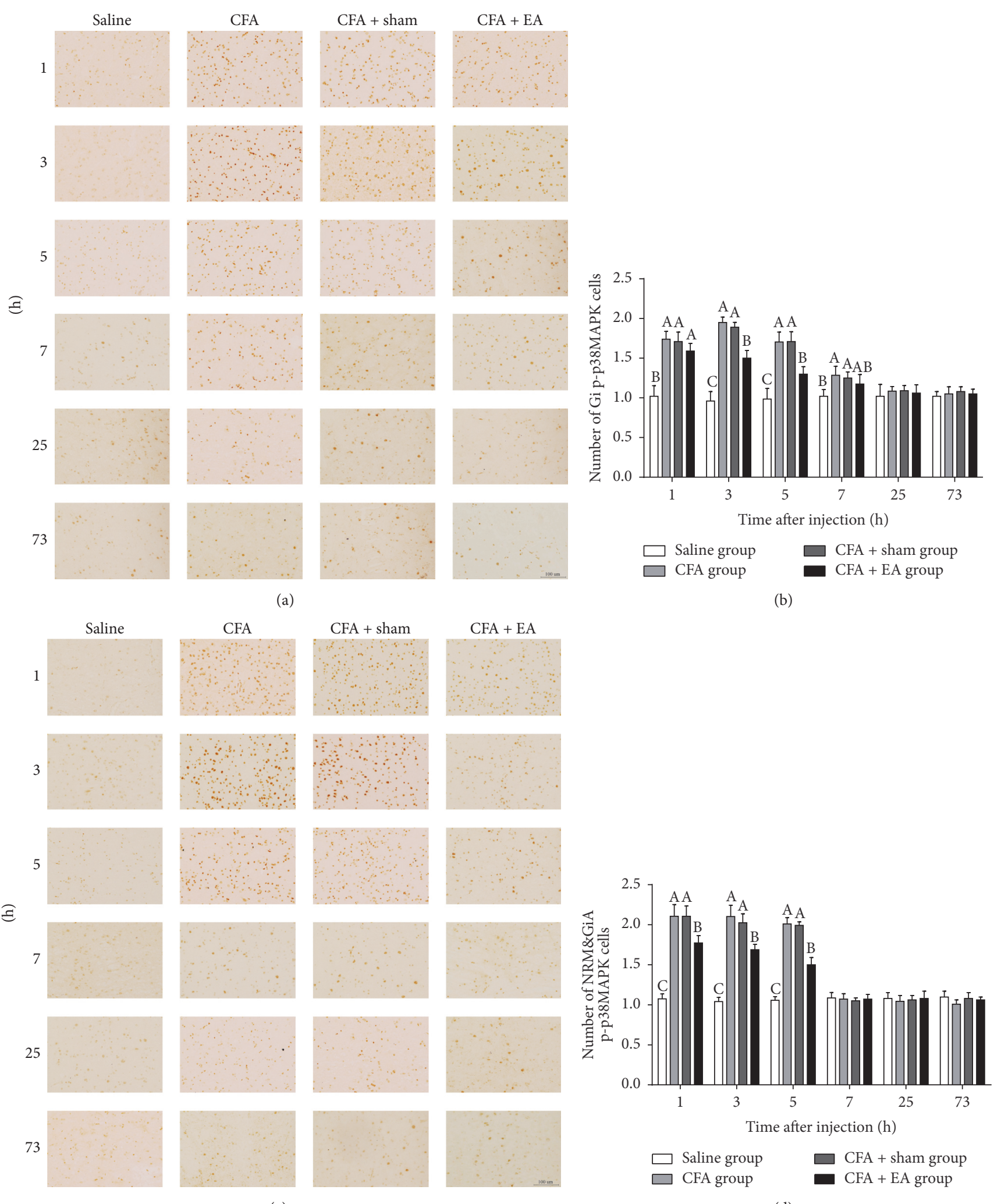

(c)

(d)

FIGURE 7: Integrated immunoreactivity analysis of p38 mitogen-activated protein kinase (p38MAPK) in the gigantocellular reticular nucleus (Gi) and the gigantocellular reticular nucleus pars alpha and the nucleus raphe magnus (GiA + NRM). Immunohistochemical staining of Phosphor-p38MAPK-Immunoreactivity (p-p38MAPK-IR) cells in Gi (a) and GiA + NRM (c) were shown in the saline, CFA, CFA + sham, and CFA + EA groups at different time points. Quantification of p-p38MAPK-IR cells showing that EA suppressed expression of p-p38MAPK-IR cells in Gi (b) and GiA + NRM (d). Values are mean \pm SD, number $\times 10^{-2} /$ field, $n=6 /$ group. The values with different letters differ significantly in the same time point $(p<0.05)$. Scale bar represents $100 \mu \mathrm{m}$. 

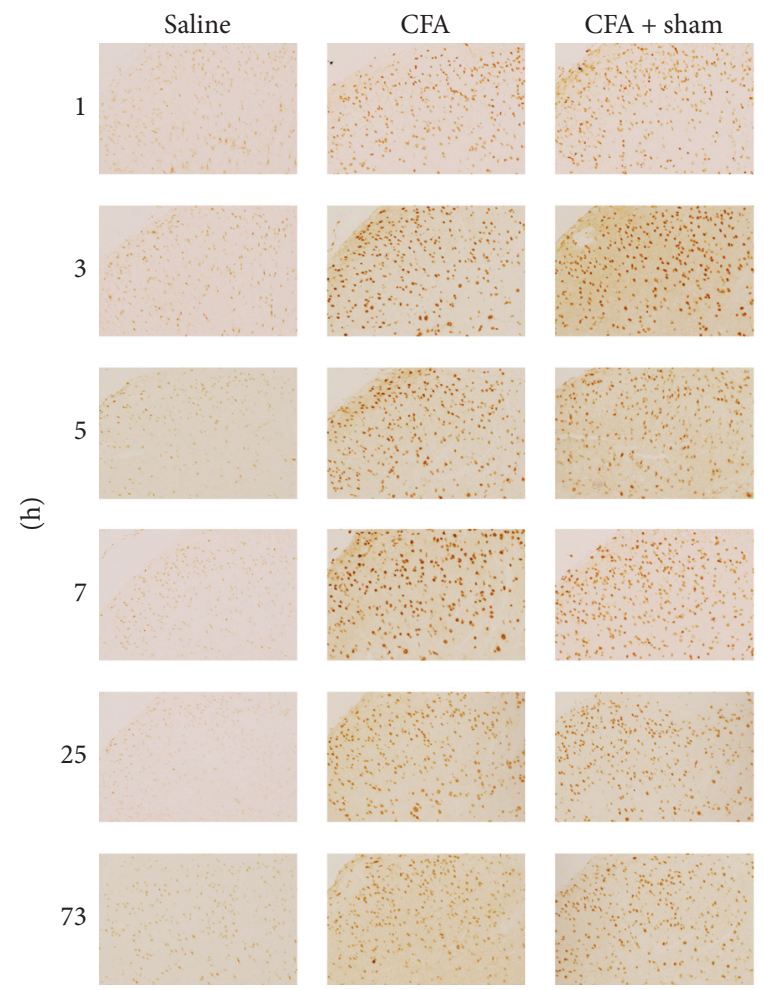

(a)
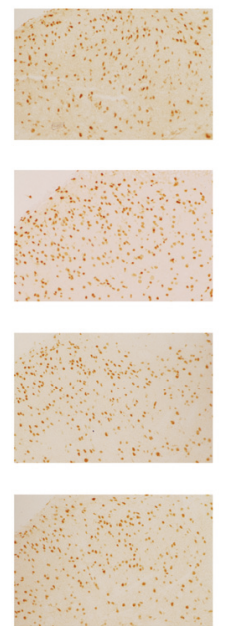
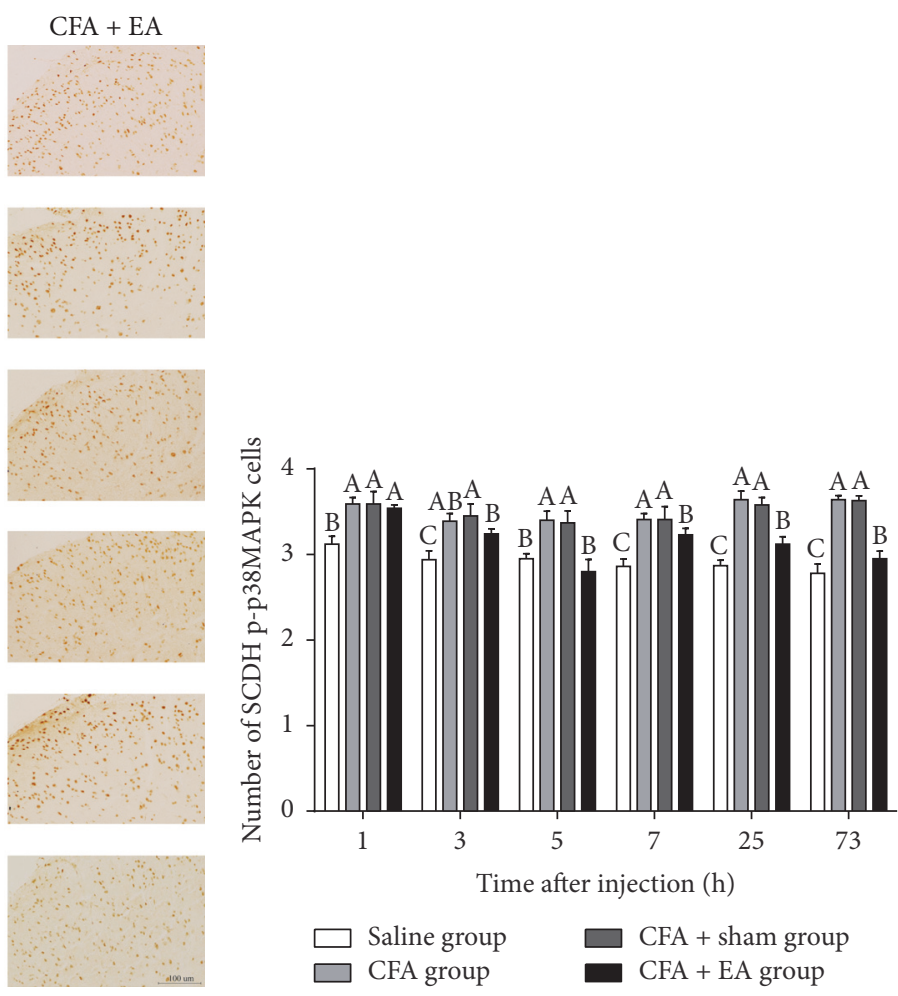

(b)

FIGURE 8: Integrated immunoreactivity analysis of p38 mitogen-activated protein kinase (p38MAPK) in the spinal cord dorsal horn (SCDH). (a) Immunohistochemical staining of Phosphor-p38MAPK-Immunoreactivity (p-p38MAPK-IR) cells in SCDH was shown in the saline, CFA, CFA + sham, and CFA + EA groups at different time points. (b) Quantification of p-p38MAPK-IR cells showing that EA suppressed expression of p-p38MAPK-IR cells in SCDH. Values are mean \pm SD, number $\times 10^{-2} /$ field, $n=6 /$ group. The values with different letters differ significantly in the same time point $(p<0.05)$. Scale bar represents $100 \mu \mathrm{m}$.

Fang et al. [29] illustrated that CFA induced an increasing number of spinal p-p38MAPK-IR cells at 3, 7, and $14 \mathrm{~d}$. Our results showed CFA induced the increasing p-p38MAPK-IR cells in the PAG and RVM, rising to a peak during $1-3 \mathrm{~h}$ and then recovering back at 5-7 h after the injection of CFA, while the number of p-p38MAPK-IR cells in SCDH increased lasting for $73 \mathrm{~h}$. Moreover, several researches provided evidence that EA exert anti-inflammatory pain by inhibiting the activation of p38MAPK in spinal level. Yi et al. [28] applied CFA into rat paw and found CFA-induced increasing of $p$ p38MAPK-IR cells was suppressed by EA at 3 and $14 \mathrm{~d}$. The same results were found in Fang et al's study [29] that EA attenuated CFA-induced inflammation pain and declined the level of spinal p-p38MAPK at 14 days after EA application. Currently, EA decreased the number of p-p38MAPK-IR cells in $\mathrm{GiA}$ and PAG at 3 and $5 \mathrm{~h}$ and in $\mathrm{Gi}+\mathrm{NRM}$ at $1-5 \mathrm{~h}$ and declined the activation of spinal p38MAPK at $3-73 \mathrm{~h}$ after CFA injection. The results suggest EA could alleviate mechanical hyperalgesia and inhibited the expression of $\mathrm{p}$ p38MAPK in PAG-RVM-SCDH.

As activated p38MAPK in spinal and supraspinal sites promotes the production of inflammatory mediators, it is possible that activated p38MAPK in the PAG, RVM, and $\mathrm{SCDH}$ can increase descending pain facilitation. Accordingly, disruption of p38MAPK or p38MAPK downstream inflammatory mediators might reverse behavioral hyperalgesia and allodynia. At supraspinal level, p-p38MAPK was localized and increased in PAG after chronic constriction nerve injury [16] and in RVM after carrageenan-induced inflammation [17]. Pretreatment with microinjection of p38MAPK inhibitor SB203580 in RVM produced a significant attenuation of behavioral hypersensitivity resulting from hind paw inflammation [9]. In the present study, EA induced antiinflammatory effect and decreased CFA-increased expression of p-p38MAPK in PAG and RVM. In addition, p38MAPK as promoter of the interleukin- $1 \beta$ (IL- $1 \beta$ ) could lead to the activation of IL- $1 \beta$ receptors [45]. Previous reports indicated that increased IL-1 $\beta$ in PAG partly mediated and enhanced pain facilitation [46]. Further, once IL- $1 \beta$ synthesis was inhibited in PAG, mechanical allodynia and thermal hyperalgesia were significantly attenuated [46]. In a carrageenan model of inflammatory pain, EA administrated at ST36 and BL60 significantly inhibited thermal hyperalgesia and carrageenanincreased mRNA expression of IL-1 receptor type I in PAG [47]. Taken together, these studies suggest that EA could attenuate inflammatory pain by disrupting activation of p38MAPK or p38MAPK downstream inflammatory mediators in RVM and PAG. 
At spinal level, the expression level of p-p38MAPK increased after peripheral inflammation which is induced by CFA [48], bee-venom [49], capsaicin [50], or formalin [20, 51]. Further, p38 inhibitor (SB20358 or SD-282) was shown to effectively attenuate hyperalgesia in peripheral tissue inflammation (intraplantar formalin or carrageenan) [20]. Fang et al. [29] found that EA at ST36 and BL60 acupoints could reduce the numbers of p-p38MAPK-IR cells in SCDH in CFA rats, and it was consistent with the present study. In addition, activated p38MAPK upregulated the synthesis of several inflammatory mediators such as cyclooxygenase-2 (COX$2)$, IL- $1 \beta$, and tumor necrosis factor- $\alpha$ (TNF $\alpha$ ) in the spinal cord to enhance central sensitization $[20,45]$, whereas these inflammatory mediators could be blocked by pretreatment with intrathecal injection of p38MAPK inhibitors [14, 52, 53]. In CFA-induced inflammatory pain model, EA could alleviate inflammatory pain behavior by downregulated COX-2 in the spinal cord [43]. CFA-increased spinal IL- $1 \beta$ and TNF- $\alpha$ were attenuated following EA treatment [54]. Taken together, these results indicate that EA may induce antihyperalgesic effect through suppressing p-p38MAPK and its downstream inflammatory mediators in SCDH in a complex mechanism.

\section{Conclusion}

EA could significantly inhibit CFA-induced decline of PWTs and CFA-induced increase of paw edema. The number of p-p38MAPK-IR cells increased in PAG, RVM, and SCDH after CFA injection, while it reduced after EA application. These results suggested that EA could disrupt descending facilitation in PAG, RVM, and SCDH and exhibits antihyperalgesic effect by inhibiting p38MAPK activation in descending facilitatory pathway.

$\begin{array}{ll}\text { Abbreviations } \\ \text { CFA: } & \text { The complete Freund adjuvant } \\ \text { COX-2: } & \text { Cyclooxygenase-2 } \\ \text { EA: } & \text { Electroacupuncture } \\ \text { Gi: } & \text { The gigantocellular reticular nucleus } \\ \text { GiA: } & \text { The gigantocellular reticular nucleus } \\ & \text { pars alpha } \\ \text { IL-1: } & \text { Interleukin-1 } \\ \text { MAPK: } & \text { Mitogen-activated protein kinase } \\ \text { NRM: } & \text { The nucleus raphe magnus } \\ \text { PAG: } & \text { The periaqueductal gray } \\ \text { p-p38MAPK: } & \text { Phosphorylated p38MAPK } \\ \text { p-p38MAPK-IR: } & \text { Phosphor-p38MAPK- } \\ & \text { Immunoreactivity } \\ \text { PWT: } & \text { Paw withdrawal threshold } \\ \text { RVM: } & \text { Rostral ventromedial medulla } \\ \text { SCDH: } & \text { Spinal cord dorsal horn } \\ \text { vlPAG: } & \text { Ventrolateral periaqueductal gray. }\end{array}$

\section{Conflicts of Interest}

All authors declare that there are no conflicts of interest.

\section{Authors' Contributions}

Man-Li $\mathrm{Hu}$ and Fei-Yan Zhou contributed equally to the work.

\section{Acknowledgments}

This work was supported by the National Natural Science Foundation of China (nos. 31672615 and 31472246).

\section{References}

[1] H. Vanegas and H.-G. Schaible, "Descending control of persistent pain: inhibitory or facilitatory?" Brain Research Reviews, vol. 46, no. 3, pp. 295-309, 2004.

[2] M. M. Behbehani and H. L. Fields, "Evidence that an excitatory connection between the periaqueductal gray and nucleus raphe magnus mediates stimulation produced analgesia," Brain Research, vol. 170, no. 1, pp. 85-93, 1979.

[3] H. Rees, M. G. Terenzi, and M. H. Roberts, “Anterior pretectal nucleus facilitation of superficial dorsal horn neurones and modulation of deafferentation pain in the rat." The Journal of Physiology, vol. 489, no. 1, pp. 159-169, 1995.

[4] H. L. Fields and A. I. Basbaum, "Brainstem control of spinal pain-transmission neurons.", Annual Review of Physiology, vol. 40, pp. 217-248, 1978.

[5] F. Porreca, M. H. Ossipov, and G. F. Gebhart, "Chronic pain and medullary descending facilitation," Trends in Neurosciences, vol. 25, no. 6, pp. 319-325, 2002.

[6] C. J. Woolf and M. W. Salter, "Neuronal plasticity: increasing the gain in pain," Science, vol. 288, no. 5472, pp. 1765-1768, 2000.

[7] M. O. Urban and G. F. Gebhart, "Supraspinal contributions to hyperalgesia," Proceedings of the National Acadamy of Sciences of the United States of America, vol. 96, no. 14, pp. 7687-7692, 1999.

[8] C. J. Garrison, P. M. Dougherty, K. C. Kajander, and S. M. Carlton, "Staining of glial fibrillary acidic protein (GFAP) in lumbar spinal cord increases following a sciatic nerve constriction injury," Brain Research, vol. 565, no. 1, pp. 1-7, 1991.

[9] J. Roberts, M. H. Ossipov, and F. Porreca, "Glial activation in the rostroventromedial medulla promotes descending facilitation to mediate inflammatory hypersensitivity," European Journal of Neuroscience, vol. 30, no. 2, pp. 229-241, 2009.

[10] H. L. Fields, "Is there a facilitating component to central pain modulation?” APS Journals, vol. 1, no. 2, pp. 71-78, 1992.

[11] L. R. Gardell, T. W. Vanderah, S. E. Gardell et al., "Enhanced evoked excitatory transmitter release in experimental neuropathy requires descending facilitation," The Journal of Neuroscience, vol. 23, no. 23, pp. 8370-8379, 2003.

[12] S. E. Burgess, L. R. Gardell, M. H. Ossipov et al., “TimeDependent Descending Facilitation from the Rostral Ventromedial Medulla Maintains, but Does Not Initiate, Neuropathic Pain," The Journal of Neuroscience, vol. 22, no. 12, pp. 5129-5136, 2002.

[13] R.-R. Ji, "Peripheral and central mechanisms of inflammatory pain, with emphasis on MAP kinases," Current Drug Targets: Inflammation \& Allergy, vol. 3, no. 3, pp. 299-303, 2004.

[14] R.-R. Ji, R. W. Gereau IV, M. Malcangio, and G. R. Strichartz, "MAP kinase and pain," Brain Research Reviews, vol. 60, no. 1, pp. 135-148, 2009. 
[15] R. M. Edelmayer, J.-D. Brederson, M. F. Jarvis, and R. S. Bitner, "Biochemical and pharmacological assessment of MAP-kinase signaling along pain pathways in experimental rodent models: a potential tool for the discovery of novel antinociceptive therapeutics," Biochemical Pharmacology, vol. 87, no. 3, pp. 390-398, 2014.

[16] H.-D. Ni, M. Yao, B. Huang et al., "Glial activation in the periaqueductal gray promotes descending facilitation of neuropathic pain through the 338 MAPK signaling pathway," Journal of Neuroscience Research, vol. 94, no. 1, pp. 50-61, 2016.

[17] H. Imbe, K. Okamoto, F. Aikawa et al., "Effects of peripheral inflammation on activation of $\mathrm{p} 38$ mitogen-activated protein kinase in the rostral ventromedial medulla," Brain Research, vol. 1134, no. 1, pp. 131-139, 2007.

[18] X. Lin, M. Wang, J. Zhang, and R. Xu, "p38 MAPK: A potential target of chronic pain," Current Medicinal Chemistry, vol. 21, no. 38, pp. 4405-4418, 2014.

[19] R. Ji and M. R. Suter, "Molecular Pain BioMed Central Review p38 MAPK, microglial signaling, and neuropathic pain".

[20] C. I. Svensson, M. Marsala, A. Westerlund et al., "Activation of p38 mitogen-activated protein kinase in spinal microglia is a critical link in inflammation-induced spinal pain processing," Journal of Neurochemistry, vol. 86, no. 6, pp. 1534-1544, 2003.

[21] E. D. Milligan, K. A. O'Connor, C. B. Armstrong et al., "Systemic administration of CNI-1493, a p38 mitogen-activated protein kinase inhibitor, blocks intrathecal human immunodeficiency virus-1 gp120-induced enhanced pain states in rats," The Journal of Pain, vol. 2, no. 6, pp. 326-333, 2001.

[22] D. M. Eisenberg, R. B. Davis, S. L. Ettner et al., "Trends in alternative medicine use in the United States, 1990-1997: results of a follow-up national survey," The Journal of the American Medical Association, vol. 280, no. 18, pp. 1569-1575, 1998.

[23] R. Zhang, L. Lao, K. Ren, and B. M. Berman, "Mechanisms of acupuncture-electroacupuncture on persistent pain," Anesthesiology, vol. 120, no. 2, pp. 482-503, 2014.

[24] J.-G. Lin and W.-L. Chen, "Acupuncture analgesia: a review of its mechanisms of actions," American Journal of Chinese Medicine, vol. 36, no. 4, pp. 635-645, 2008.

[25] Z.-Q. Zhao, "Neural mechanism underlying acupuncture analgesia," Progress in Neurobiology, vol. 85, no. 4, pp. 355-375, 2008.

[26] F. Luo and J.-Y. Wang, "Modulation of central nociceptive coding by acupoint stimulation," Neurochemical Research, vol. 33, no. 10, pp. 1950-1955, 2008.

[27] V. V. Romita, A. Suk, and J. L. Henry, "Parametric studies on electroacupuncture-like stimulation in a rat model: effects of intensity, frequency, and duration of stimulation on evoked antinociception," Brain Research Bulletin, vol. 42, no. 4, pp. 289296, 1997.

[28] Y. Liang, J. Q. Fang, J. Y. Du, and J. F. Fang, "Effect of electroacupuncture on activation of p38MAPK in spinal dorsal horn in rats with complete Freund's adjuvant-induced inflammatory pain," Evidence-Based Complementary and Alternative Medicine, vol. 2012, Article ID 568273, 6 pages, 2012.

[29] J.-Q. Fang, J.-Y. Du, Y. Liang, and J.-F. Fang, "Intervention of electroacupuncture on spinal p38 MAPK/ATF-2/VR-1 pathway in treating inflammatory pain induced by CFA in rats," Molecular Pain, vol. 9, no. 1, article 13, 2013.

[30] R. Minfeng and H. Jisheng, "Rat tail flick acupuncture analgesia model," Chinese Medical Journal, vol. 92, no. 8, pp. 576-582, 1979.
[31] L. Cui, Y. Ding, Y. Feng et al., "MiRNAs are involved in chronic electroacupuncture tolerance in the rat hypothalamus," Molecular Neurobiology, vol. 54, no. 2, pp. 1429-1439, 2017.

[32] Y.-Q. Zhang, G.-C. Ji, G.-C. Wu, and Z.-Q. Zhao, "Excitatory amino acid receptor antagonists and electroacupuncture synergetically inhibit carrageenan-induced behavioral hyperalgesia and spinal fos expression in rats," PAIN, vol. 99, no. 3, pp. 525$535,2002$.

[33] C. Watson, The Rat Brain in Stereotaxic Coordinates-The New Coronal Set, Academic press, 2004.

[34] K. Ren and R. Dubner, "Inflammatory models of pain and hyperalgesia," ILAR Journal, vol. 40, no. 3, pp. 111-118, 1999.

[35] M. J. Iadarola, L. S. Brady, G. Draisci, and R. Dubner, "Enhancement of dynorphin gene expression in spinal cord following experimental inflammation: stimulus specificity, behavioral parameters and opioid receptor binding," PAIN, vol. 35, no. 3, pp. 313-326, 1988.

[36] W. Guo, S. Zou, Y. Guan et al., "Tyrosine phosphorylation of the NR2B subunit of the NMDA receptor in the spinal cord during the development and maintenance of inflammatory hyperalgesia," The Journal of Neuroscience, vol. 22, no. 14, pp. 6208-6217, 2002.

[37] K. Ren, J. L. K. Hylden, G. M. Williams, M. A. Ruda, and R. Dubner, "The effects of a non-competitive NMDA receptor antagonist, MK-801, on behavioral hyperalgesia and dorsal horn neuronal activity in rats with unilateral inflammation," PAIN, vol. 50, no. 3, pp. 331-344, 1992.

[38] J. L. K. Hylden, R. L. Nahin, R. J. Traub, and R. Dubner, "Expansion of receptive fields of spinal lamina I projection neurons in rats with unilateral adjuvant-induced inflammation: the contribution of dorsal horn mechanisms," PAIN, vol. 37, no. 2, pp. 229-243, 1989.

[39] J.-S. Han, "Acupuncture and endorphins," Neuroscience Letters, vol. 361, no. 1-3, pp. 258-261, 2004.

[40] J. S. Han, "Acupuncture: neuropeptide release produced by electrical stimulation of different frequencies," Trends in Neurosciences, vol. 26, no. 1, pp. 17-22, 2003.

[41] C.-P. Huang, H.-N. Chen, H.-L. Su et al., "Electroacupuncture reduces carrageenan- and CFA-induced inflammatory pain accompanied by changing the expression of Nav1.7 and Nav1.8, rather than Nav1.9, in mice dorsal root ganglia," Evidence-Based Complementary and Alternative Medicine, vol. 2013, Article ID 312184, 8 pages, 2013.

[42] Y.-J. Zeng, S.-Y. Tsai, K.-B. Chen, S.-F. Hsu, J. Y.-R. Chen, and Y.-R. Wen, "Comparison of electroacupuncture and morphinemediated analgesic patterns in a plantar incision-induced pain model," Evidence-Based Complementary and Alternative Medicine, vol. 2014, Article ID 659343, 12 pages, 2014.

[43] J.-Y. Du, J.-Q. Fang, Y. Liang, and J.-F. Fang, "Electroacupuncture attenuates mechanical allodynia by suppressing the spinal JNK1/2 pathway in a rat model of inflammatory pain," Brain Research Bulletin, vol. 108, pp. 27-36, 2014.

[44] J. Q. Fang, J. F. Fang, Y. Liang, and J. Y. Du, "Electroacupuncture mediates extracellular signal-regulated kinase 1/2 pathways in the spinal cord of rats with inflammatory pain," $B M C$ Complementary \& Alternative Medicine, vol. 14, no. 1, article 285, 2014.

[45] R. R. Ji and M. R. Suter, "p38 MAPK, microglial signaling, and neuropathic pain," Molecular Pain, vol. 3, article 33, 2007.

[46] H. Chu, J. Sun, H. Xu, Z. Niu, and M. Xu, "Effect of periaqueductal gray melanocortin 4 receptor in pain facilitation 
and glial activation in rat model of chronic constriction injury," Neurological Research, vol. 34, no. 9, pp. 871-888, 2012.

[47] G. C. Ji, J. Yu, Z. Q. Dong, and G. C. Wu, "Changes of Expression of IL-1 Receptor-I mRNA in Rat Periaqueductal Gray after Peripheral Inflammation and Electroacupuncture Analgesia," Acupuncture Research, 2003.

[48] B. DL, J. TL, and H. D, "Regulation of peripheral inflammation by spinal p38 MAP kinase in rats," in Plos Medicine, vol. 3, p. e338, PLoS Medicine, 9 edition, 2006.

[49] X.-Y. Cui, Y. Dai, S.-L. Wang et al., "Differential activation of p38 and extracellular signal-regulated kinase in spinal cord in a model of bee venom-induced inflammation and hyperalgesia," Molecular Pain, vol. 4, article no. 17, 2008.

[50] S. M. Sweitzer, M. C. Peters, J. Y. Ma et al., "Peripheral and central p38 MAPK mediates capsaicin-induced hyperalgesia," PAIN, vol. 111, no. 3, pp. 278-285, 2004.

[51] S.-Y. Kim, J.-C. Bae, J.-Y. Kim et al., "Activation of p38 MAP kinase in the rat dorsal root ganglia and spinal cord following peripheral inflammation and nerve injury," NeuroReport, vol. 13, no. 18, pp. 2483-2486, 2002.

[52] C.-S. Sung, Z.-H. Wen, W.-K. Chang et al., "Inhibition of p38 mitogen-activated protein kinase attenuates interleukin- $1 \beta$ induced thermal hyperalgesia and inducible nitric oxide synthase expression in the spinal cord," Journal of Neurochemistry, vol. 94, no. 3, pp. 742-752, 2005.

[53] J. Saklatvala, "The p38 MAP kinase pathway as a therapeutic target in inflammatory disease," Current Opinion in Pharmacology, vol. 4, no. 4, pp. 372-377, 2004.

[54] W.-L. Mi, Q.-L. Mao-Ying, X.-W. Wang et al., "Involvement of spinal neurotrophin-3 in electroacupuncture analgesia and inhibition of spinal glial activation in rat model of monoarthritis," The Journal of Pain, vol. 12, no. 9, pp. 974-984, 2011. 


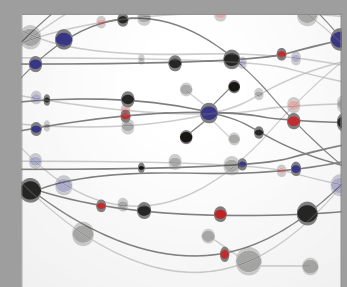

The Scientific World Journal
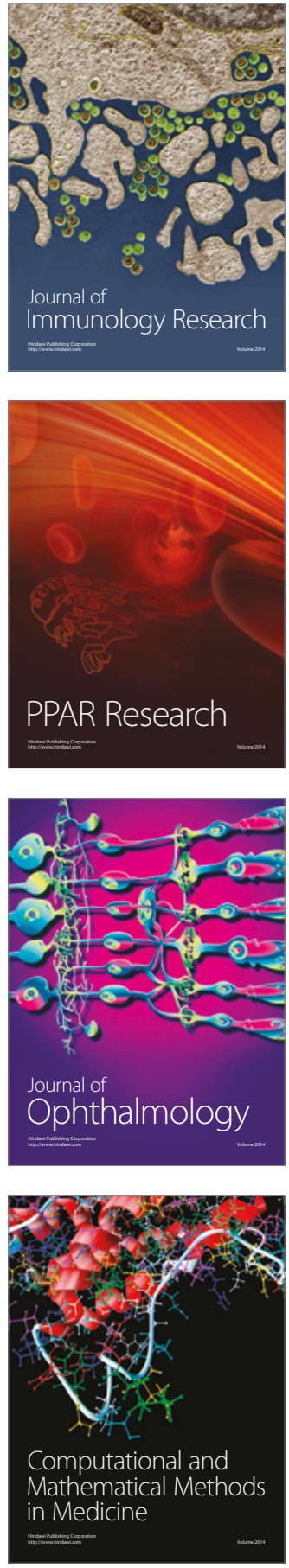

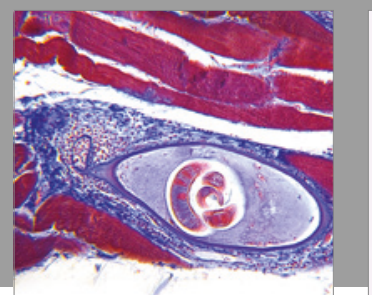

Gastroenterology Research and Practice
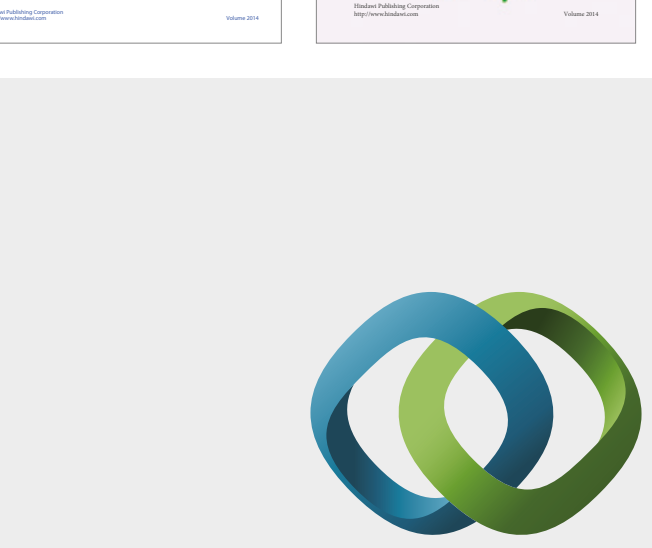

\section{Hindawi}

Submit your manuscripts at

https://www.hindawi.com
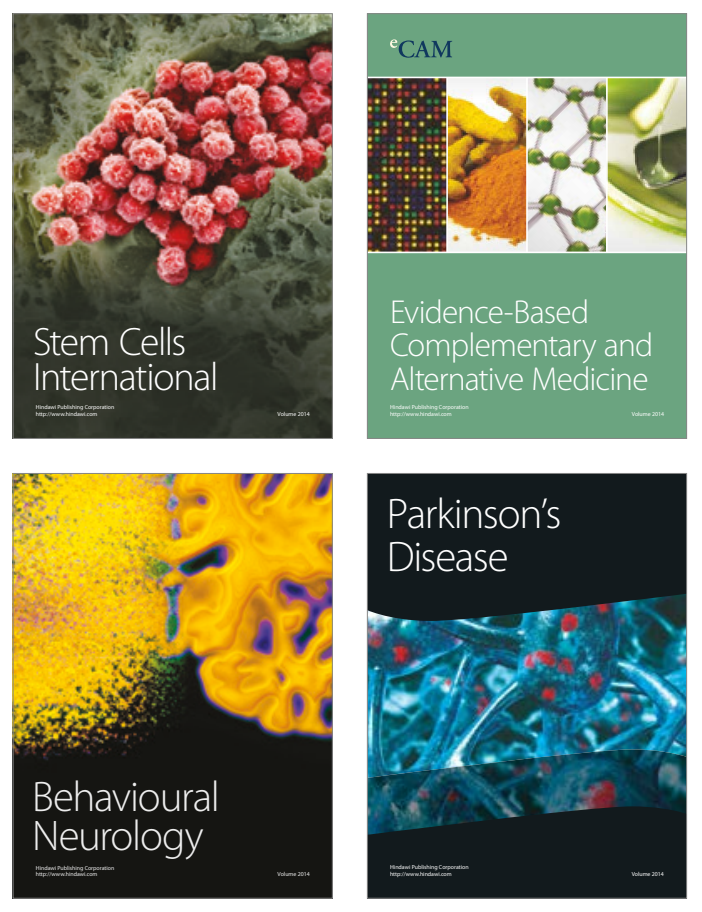
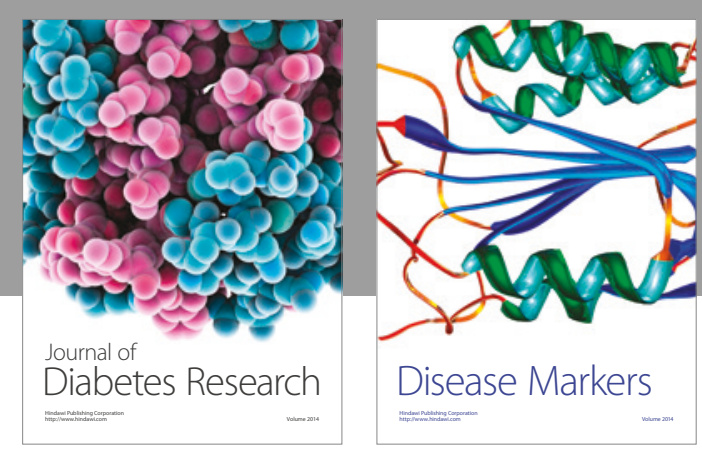

Disease Markers
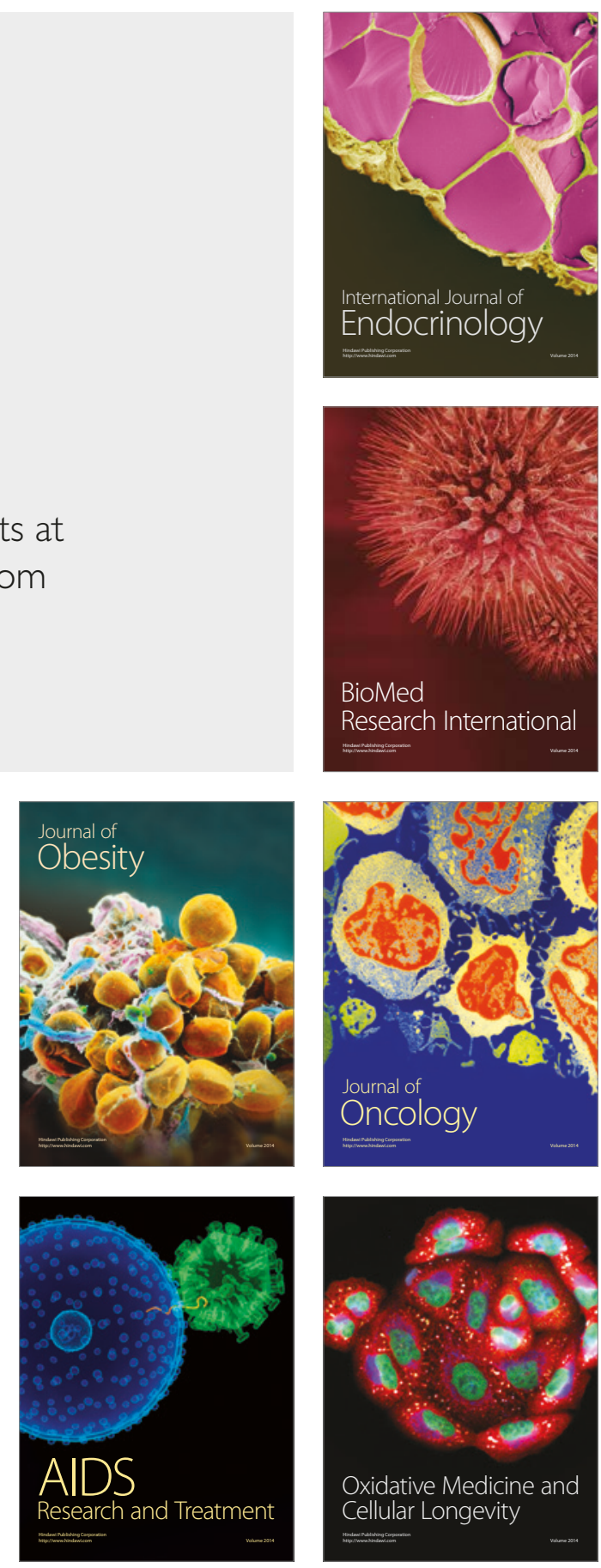